\title{
Focal and Temporal Release of Glutamate in the Mushroom Bodies Improves Olfactory Memory in Apis mellifera
}

\author{
Fernando Locatelli, ${ }^{1}$ Gesine Bundrock, ${ }^{1}$ and Uli Müller ${ }^{1,2}$ \\ ${ }^{1}$ Freie Universität Berlin, Institut für Biologie, Neurobiologie, D-14195 Berlin, Germany, and 2Naturwissenschaftlich-Technische Fakultät III, Fachrichtung \\ 8.3, Biowissenschaften, Zoologie/Physiologie, Universität des Saarlandes, D-66041 Saarbrücken, Germany
}

In contrast to vertebrates, the role of the neurotransmitter glutamate in learning and memory in insects has hardly been investigated. The reason is that a pharmacological characterization of insect glutamate receptors is still missing; furthermore, it is difficult to locally restrict pharmacological interventions. In this study, we overcome these problems by using locally and temporally defined photo-uncaging of glutamate to study its role in olfactory learning and memory formation in the honeybee, Apis mellifera. Uncaging glutamate in the mushroom bodies immediately after a weak training protocol induced a higher memory rate $2 \mathrm{~d}$ after training, mimicking the effect of a strong training protocol. Glutamate release before training does not facilitate memory formation, suggesting that glutamate mediates processes triggered by training and required for memory formation. Uncaging glutamate in the antennal lobes shows no effect on memory formation. These results provide the first direct evidence for a temporally and locally restricted function of glutamate in memory formation in honeybees and insects.

Key words: learning; memory; glutamate; caged glutamate; insects; Apis mellifera

\section{Introduction}

L-Glutamate is the major excitatory neurotransmitter in the vertebrate brain; the roles played by the various glutamate receptors in learning and memory are established (Riedel et al., 2003). As in vertebrates, the critical role of glutamate receptors on the induction and stabilization of neural plasticity was demonstrated in the marine snail Aplysia (Roberts and Glanzman, 2003). In insects, the study of glutamatergic neurotransmission has been mainly focused on its role in the neuromuscular junction (Jan and Jan, 1976). There is, however, considerable evidence indicating that glutamate also acts as a neurotransmitter in the insect CNS. Glutamate-like immunoreactivity (Bicker et al., 1988; Schürmann et al., 2000; Sinakevitch et al., 2001) and glutamateinduced ion currents (Cayre et al., 1999) were described in insect CNS neurons. Moreover, molecular studies identified the expression of glutamate transporters and glutamate receptors homologous to mammalian ones (Ultsch et al., 1993; Parmentier et al., 1996; Seal et al., 1998; Kucharski et al., 2000; Volkner et al., 2000; Funada et al., 2004).

The limited knowledge about the function of glutamate in insect behavior is mainly attributable to lacking pharmacological characterization of insect glutamate receptors and the difficulty involved in locally restricting pharmacological interventions. Hence, previous works aimed at studying the role of glutamate in

Received July 29, 2005; revised Nov. 7, 2005; accepted Nov. 7, 2005.

This work was supported by the Deutsche Forschungsgemeinschaft (Sonderforschungsbereich 515). F.L. was supported by the German Academic Exchange Service and Fundación Antorchas, Argentina. We thank Drs. R. Menzel and $\mathrm{G}$. Leboulle for helpful discussions and M. Wurm for help with this manuscript.

Correspondence should be addressed to Fernando Locatelli, Freie Universität Berlin, Institut für Biologie, Neurobiologie, Königin-Luise-Strasse 28/30, D-14195 Berlin, Germany. E-mail: locatellif@yahoo.com.ar.

DOI:10.1523/JNEUROSCI.3180-05.2005

Copyright $\odot 2005$ Society for Neuroscience ～0270-6474/05/2511614-05\$15.00/0 the insect brain were based on systemic administration of drugs, whose action specificities are described in vertebrates (Jaffe and Blanco, 1994; Lopatina et al., 2002; Si et al., 2004).

Recently, molecular genetic tools provided evidence that the NMDA glutamate receptors (NMDARs) are involved in learning and memory in Drosophila (Xia et al., 2005). Stable and transient knockdown of NMDARs in the whole brain disrupts olfactory learning and impairs long-term memory. However, the particular brain site at which glutamate influences memory remains unknown.

In this work, we investigated the function of glutamate on learning and memory in the olfactory learning paradigm of the honeybee, Apis mellifera. In this paradigm, the animals can be trained and behavior monitored while the head capsule is open and the brain is exposed. This condition makes the brain accessible for locally and temporally defined release of glutamate by photolytic stimulation of caged glutamate (Wieboldt et al., 1994). We demonstrate that photorelease of glutamate in the mushroom bodies (MBs) facilitates olfactory memory. The results provide evidence that glutamatergic neurotransmission in the mushroom bodies is involved in memory formation.

\section{Materials and Methods}

Animals. Honeybees (A. mellifera) were caught at the hive entrance, cooled, and restrained in harnesses (Bitterman et al., 1983). A window was cut into the head capsule between the antennae and the ocelli, allowing visual access to the brain. Tracheae and neurolemma covering the brain were left intact. Bees with glands obstructing a clear view of the brain were omitted from the experiments. All experiments started $1 \mathrm{~d}$ after this manipulation. Every evening the bees were fed to satiation with sucrose solution $30 \% \mathrm{w} / \mathrm{v}$. Animals were kept overnight in a dark humid box at room temperature.

Photolysis of caged glutamate. $\gamma$-(CNB-caged) L-glutamic acid 
[L-glutamic acid, $\gamma$-( $\alpha$-carboxy-2-nitrobenzyl) ester, trifluoroacetic acid salt] and $\alpha$-(DMNB-caged) L-glutamic acid [L-glutamic acid, $\alpha$-(4,5dimethoxy-2-nitrobenzyl) ester, hydrochloride] were purchased from Invitrogen (Groningen, The Netherlands). Photolytic illumination was provided by a UV Flash lamp (T.I.L.L. Photonics, Martinsreid, Germany) that produced flashes between 340 and $390 \mathrm{~nm}$ wavelength, $0.5 \mathrm{~ms}$ duration, and $10^{15}$ photons $/ \mathrm{mm}^{2}$ in the back focal plane of the microscope. The flash output entered the photo-adapter port [Olympus (Tokyo, Japan) SZ-CTV] of a binocular (Olympus SZ40). A mask with two apertures in the plane of focus provided two simulation spots in the specimen plane of the binocular.

In the experiments in which glutamate release was evaluated by photostimulating caged glutamate in vitro and in situ, a drop of caged compound solution or the dissected brain was placed on a cover glass and stimulated with five UV light flashes. In the behavioral experiments, animals were positioned below the binocular with the brain in its specimen plane. The position of the animal and the mask defining the illumination spots allowed discrete photostimulation of the desired brain regions (Müller, 2000).

Behavioral experiments. All behavioral assays were performed between 12:00 P.M. and 3:00 P.M. from June to September, 2004, in Berlin, Germany. Twenty minutes before the training, animals received injections of either $1 \mu \mathrm{l}$ of vehicle [5\% DMSO in PBS (in mM: $137 \mathrm{NaCl}, 2.7 \mathrm{KCl}, 10.1$ $\mathrm{Na}_{2} \mathrm{HPO}_{4}$, and $\left.\left.1.8 \mathrm{KH}_{2} \mathrm{PO}_{4}, \mathrm{pH} 7.2\right)\right]$ or $1 \mu \mathrm{l}$ of vehicle containing a mixture of CNB- and DMNB-caged glutamate (1 mM each) into the hemolymph in the thorax.

One minute before training, animals were put under the binocular, where training and photostimulation were performed. Training consisted of a single-trial olfactory conditioning of the proboscis extension response (PER), in which an odor (carnation) served as the conditioned stimulus (CS), and sucrose solution $(20 \% \mathrm{w} / \mathrm{v})$ served as the unconditioned stimulus (US) (Bitterman et al., 1983). Three seconds after onset of the CS ( $5 \mathrm{~s}$ duration), the US was applied to the antennae. When the bee extended the proboscis, it was allowed to lick the solution for $3 \mathrm{~s}$. Between 0 and $5 \%$ of all of the animals prepared for the experiment showed no response to the US and were excluded from the experiment. Thirty seconds after the end of the training trial, the animal was moved to its resting position.

Extension of the proboscis beyond the virtual line between the open mandibles during CS presentation was counted as a positive response. Statistical analysis was based on ANOVA for repeated measurements. When the retention curves were statistically different between groups, a $\chi^{2}$ test was performed for each testing trial.

Determination of caged glutamate photolysis. L-glutamic acid was measured by ELISA using antibodies that recognize glutamateglutardialdehyde conjugates (Sigma-Aldrich Laborchemikalien, Seelze, Germany). The photo-uncaging of glutamate in vitro was evaluated by photostimulating a solution of CNB-caged glutamate, which is not recognized by the antibody. Ten microliters of a solution of $1 \mathrm{~mm}$ caged glutamate, photostimulated or not, were incubated with $2 \mu \mathrm{l}$ of $25 \%$ glutardialdehyde and $10 \mu \mathrm{g}$ of BSA in a volume of $100 \mu \mathrm{l}$ of PBS for $1 \mathrm{~h}$ at $4^{\circ} \mathrm{C}$. The same reaction was performed with defined amounts of glutamate. The reactions were stopped with $100 \mu \mathrm{l}$ of $100 \mathrm{~mm}$, Tris pH 7.5. Glutamate-like immunoreactivity was determined following standard ELISA protocols (Crowther, 2001).

To corroborate the photolysis of caged glutamate in situ, animals were injected $1 \mathrm{~h}$ before the photostimulation with the glutamate reuptake blocker L-trans-2,4-PDC (Tocris Cookson, Bristol, UK) and $20 \mathrm{~min}$ before photostimulation with $1 \mu \mathrm{l}$ of $10 \mathrm{~mm} \mathrm{CNB}$-caged glutamate. Immediately before photostimulation, central brains were dissected, briefly rinsed in PBS and placed on a cover glass where the brains were either photostimulated or not. Immediately after stimulation, the brains were homogenized in $1 \mathrm{ml}$ (PBS, 2 mm EGTA, 2 mm EDTA). Samples of $400 \mu \mathrm{l}$ of crude homogenate were incubated $1 \mathrm{~h}$ with $8 \mu \mathrm{l}$ of $25 \%$ glutardialdehyde and $10 \mu \mathrm{g}$ of BSA. The reactions were stopped with $400 \mu \mathrm{l}$ of 100 $\mathrm{mm}$, Tris $\mathrm{pH}$ 7.5. The glutamate concentration in the mixture was determined by ELISA. The signal-to-noise ratio of the measurements was considerably improved by preincubation of the antiglutamate antibody with GABA-, glutamine-, aspartate-, alanine-, and taurine-
A

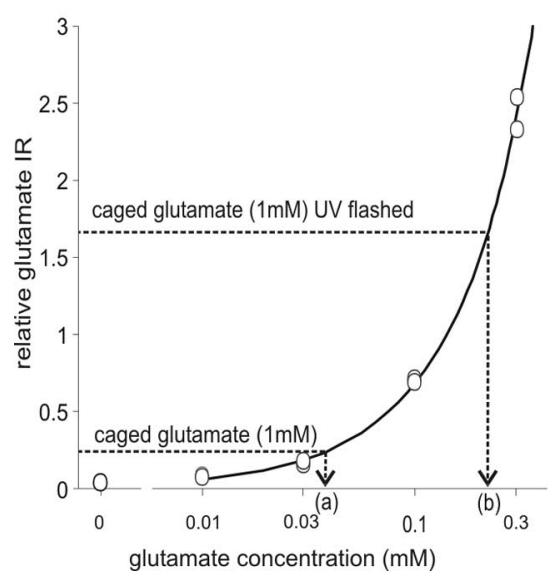

B

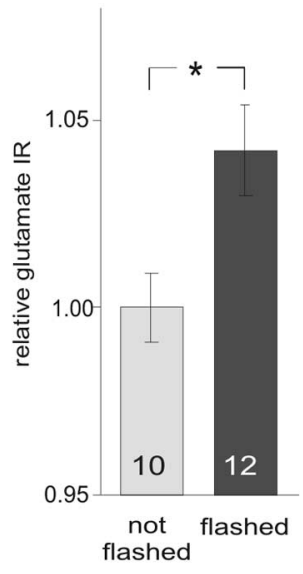

Figure 1. Photo-uncaging of glutamate determined by glutamate-like immunoreactivity. $\boldsymbol{A}$, Relative glutamate immunoreactivity determined by ELISA in samples containing defined concentrations of L-glutamate $(\bigcirc)$ or $1 \mathrm{~mm} \gamma$-(CNB-caged) L-glutamate, photostimulated (b) or not (a). The curve was determined using duplicate samples. Fitted curve: $y=5.9492 x^{2}+$ $6.3389 x-0.0078 ; R^{2}=0.9967$. The amount of uncaged glutamate was calculated using the obtained curve and represents the mean of four determinations each. $\boldsymbol{B}$, Uncaging of glutamate in situ. The bees were given systemic injection of $1 \mu \mathrm{l}$ of $10 \mathrm{~mm} \gamma$-(CNB-caged) L-glutamate. Twenty minutes later, the brains were dissected and treated (or not) with five UV flashes. Glutamate immunoreactivity in brain extracts is shown normalized to the average of nonphotostimulated brains. Numbers in the bars represent the number of animals. ${ }^{*} p<0.05$ ( $t$ test). IR, Immunoreactivity.

glutardialdehyde conjugates. The preabsorbed antibody was used in a dilution 1:10,000 in PBS and 1\% BSA.

\section{Results}

\section{Photo-uncaging of L-glutamate}

As a prerequisite for the present work, we confirmed the effectiveness of a photostimulation protocol used to induce photolysis of caged glutamate in vivo. The photolysis of caged glutamate and consequent release of glutamate was measured by an ELISA using antibodies that recognize glutamate-glutardialdehyde conjugates but not caged glutamate conjugates.

In the first experiment, the photostimulation conditions necessary to induce photorelease of glutamate were tested in vitro. A solution containing $1 \mathrm{~mm} \gamma$-CNB-caged glutamate was split, and one-half was illuminated with UV light flashes. The glutamatelike immunoreactivity was measured in both solutions and in standard solutions containing defined concentrations of L-glutamate (Fig. 1A). A $1 \mathrm{~mm}$ concentration of caged glutamate (non-photostimulated) yields a glutamate immunoreactivity equivalent to $36 \pm 2 \mu \mathrm{M}$ glutamate, most likely attributable to a small amount of free glutamate in the caged glutamate solution. The photostimulation of $1 \mathrm{~mm}$ caged glutamate leads to a signal equivalent to $217 \pm 6 \mu \mathrm{M}$ of glutamate. This result, under these experimental conditions, indicates a glutamate photorelease of $\sim 20 \%$ of the caged glutamate used.

The next experiment was performed to confirm the ability of the photostimulation protocol to induce glutamate uncaging in situ. The honeybees received a systemic injection of caged glutamate into the thorax; glutamate-like immunoreactivity was measured in brain homogenates prepared immediately after photostimulation. An increase of glutamate in photostimulated brains versus nonstimulated brains confirms the two requirements needed for the present work: first, the caged compound reaches the brain after injection into the thorax; second, the photostimu- 
lation protocol is effective at inducing photolysis of the caged compound in situ. The detection of the uncaged glutamate in the brain could be masked by the high endogenous glutamate concentration and the rapid recycling of glutamate by the endogenous reuptake mechanisms. To minimize these effects, the animals were preinjected with the glutamate reuptake blocker L-trans-2,4-PDC ( $1 \mu \mathrm{l} ; 50 \mathrm{~mm})$, and a higher amount of caged glutamate was injected $(1 \mu \mathrm{l} ; 10 \mathrm{~mm})$. In addition, the time between photostimulation and homogenization and thus the fast decay of photoreleased glutamate was further reduced by dissecting the brains before photostimulation. This procedure reduced the time between photostimulation and homogenization to only $5 \mathrm{~s}$. The small but significant increase in glutamate immunoreactivity observed in the photostimulated brains indicates the ability of the method to induce glutamate uncaging in situ (Fig. $1 \mathrm{~B}$ ).

\section{Uncaging of L-glutamate in the mushroom bodies improves memory}

The mushroom bodies are major sites mediating olfactory learning and memory in insects (Heisenberg et al., 1985; de Belle and Heisenberg, 1994; Connolly et al., 1996; Hammer and Menzel, 1998). Furthermore, the critical role of specific subregions of the mushroom bodies in long-term memory (LTM) was demonstrated recently (Pascual and Preat, 2001; Isabel et al., 2004). This and the evidence of glutamatergic neurotransmission in this brain structure (Cayre et al., 1999; Schürmann et al., 2000) prompted us to investigate the role of glutamate in learning and memory directing the photorelease of glutamate to this particular brain region. Because photolysis of caged compounds leads to an increase of the compound concentration in the millisecond-tosecond range, it provides a perfect tool for evaluating the function of glutamate in the temporal domain.

All experiments were based on comparisons between animals that received the same training and photostimulation protocols but had been injected previously with either vehicle or caged glutamate.

In the first experiment, we tested the effect of releasing glutamate in the mushroom bodies $1 \mathrm{~min}$ before training (Fig. $2 \mathrm{~A}$ ). Two illumination spots of $400 \mu \mathrm{m}$ diameter provided simultaneous bilateral photostimulation of the lateral and median calyces, which constitute the input area of the olfactory and the reward pathways into the mushroom bodies. As observed in Figure $2 \mathrm{~A}$, glutamate release before training affected neither the spontaneous responsiveness to the odor nor the unconditioned response to sugar solution during the training, nor does it affect memory performance.

In the next experiment, we tested whether glutamate release immediately after training affects memory formation (Fig. 2 B). Analysis of the retention curve revealed significant differences between both groups (ANOVA repeated measurements; $F=$ $4.85 ; p<0.05)$. Although memory tested $2 \mathrm{~h}$ after training was unaffected by posttraining release of glutamate $\left(\chi^{2}=0.26 ; p=\right.$ $0.611)$, memory tested at day 1 and day 2 was increased. Comparison of the conditioned PER $1 \mathrm{~d}\left(\chi^{2}=3.31 ; p=0.068\right)$ and $2 \mathrm{~d}$ after training $\left(\chi^{2}=6.87 ; p=0.008\right)$ reveals a critical function for glutamate in the mushroom bodies regarding the induction of a late memory stage.

Because the antennal lobes are actively involved in olfactory memory formation (Müller, 2000), we tested whether glutamate released in the antennal lobes also affects memory formation. Two stimulation spots of $\sim 250 \mu \mathrm{m}$ diameter each were used to induce bilateral glutamate-uncaging in the antennal lobes immediately after training.
A

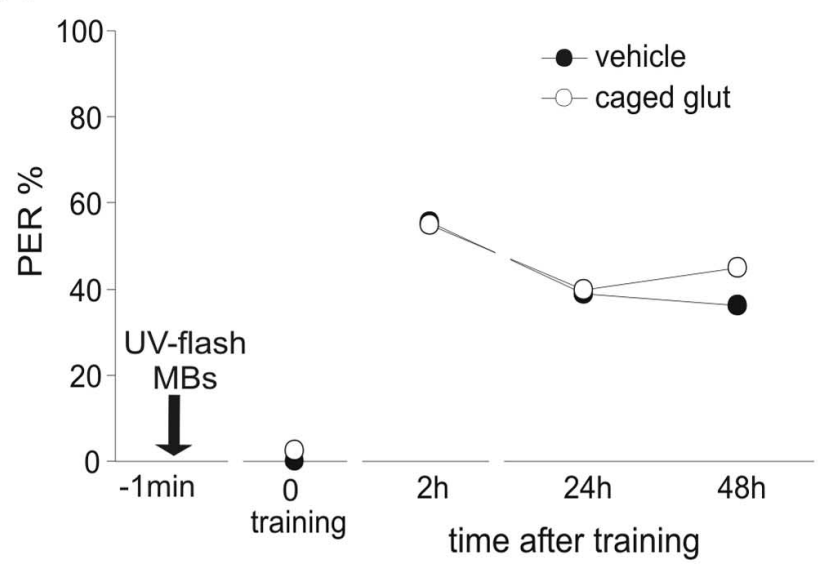

B
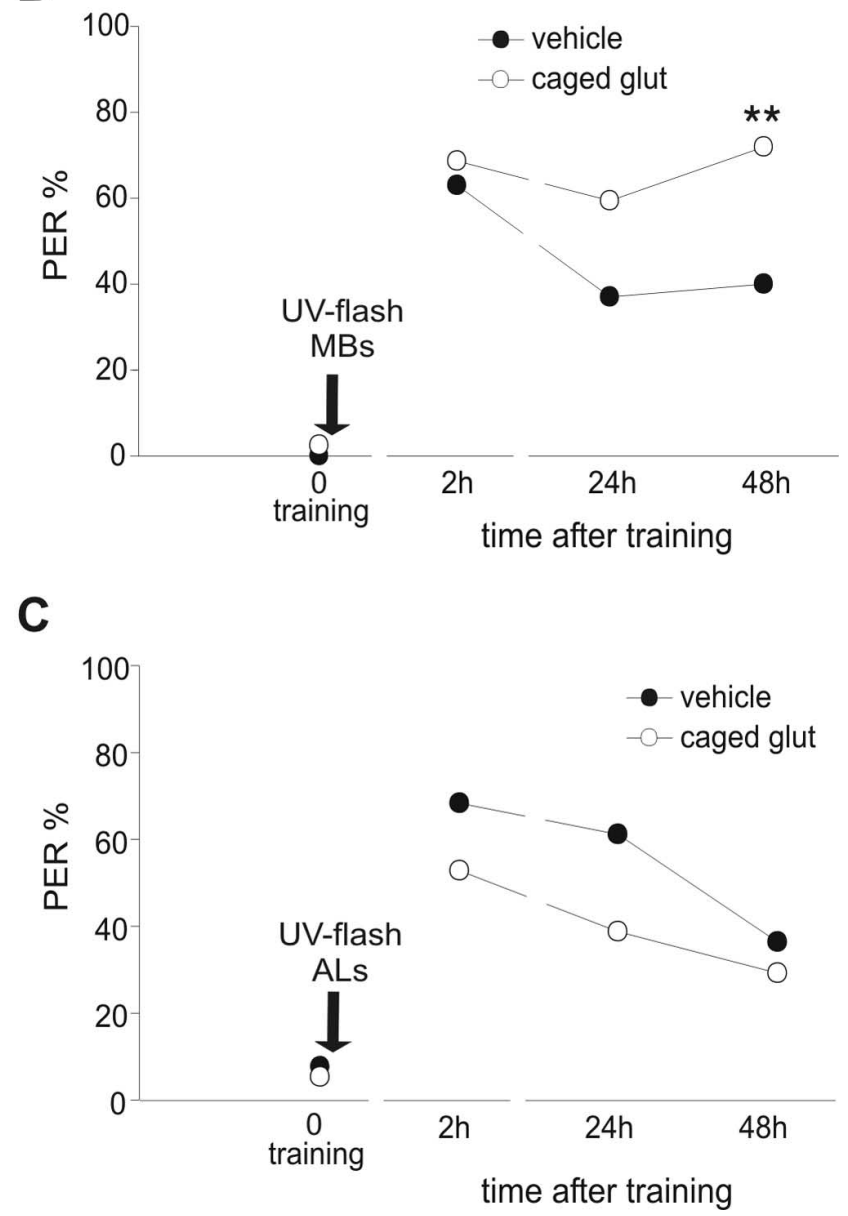

Figure 2. Effects of temporally and locally defined photo-uncaging of glutamate on honeybee olfactory memory. Twenty minutes before training, the bees received systemic injections of vehicle or caged glutamate. The arrows indicate the site and time ( $1 \mathrm{~min}$ before or $5 \mathrm{~s}$ after training) of photostimulation (UV flash). Each data point represents the probability of PER elicited by odor during training and testing sessions. A, Photorelease of glutamate in the mushroom bodies 1 min before training does not affect learning or memory. Vehicle, $n=36$; caged glutamate, $n=40$ (ANOVA repeated measurements, $F=0.14 ; p=0.71$ ). $\boldsymbol{B}$, Photorelease of glutamate in the mushroom bodies immediately after training improves LTM. Vehicle, $n=35$; caged glutamate, $n=32$ (ANOVA repeated measurements, $F=4.86 ; p=0.03$ between groups and post hoc test; $\chi^{2}$ test, $\left.{ }^{* *} p<0.01\right)$. C, Photorelease of glutamate in the antennal lobes (ALs) immediately after training does not affect learning or memory. Vehicle, $n=28$; caged glutamate, $n=21$ (ANOVA repeated measurements: $F=1.87 ; p=0.18)$. glu, Glutamate. 
As shown in Figure 2C, no statistical differences were found between the groups. However, the observation that memory performance after uncaging glutamate in the antennal lobes is worse than that in the control group may point to an inhibitory role of glutamate in the antennal lobes compared with the mushroom bodies. This result indicates a clear difference of glutamate function in the mushroom bodies and the antennal lobes.

\section{Discussion}

Light-induced uncaging of glutamate was used previously to study the structure and excitability of dendritic trees and to map glutamate receptors in brain slices and cultured cells (for review, see Callaway and Yuste, 2002). Our present work demonstrates that this technique is also applicable to manipulate and study glutamatergic neurotransmission in vivo. Compared with local pressure application using microcapillaries, light stimulation is relatively innocuous and thus very advantageous for in vivo studies.

The determination that caged glutamate can be photolyzed in vivo to induce an increase in glutamate concentration is qualitative and does not permit us to know the concentration of glutamate reached by the photostimulation (Fig. $1 B$ ). The effectiveness of the glutamate uncaging is, however, confirmed at the behavioral level (Fig. 2). As a whole, the experiments reported here confirm that the effect observed on memory is specific for the photoinduced glutamate uncaging, and they rule out an alternative explanation in terms of the caged compound itself or spontaneous uncaging of glutamate. If the latter arguments are correct, all animals injected with caged glutamate should show enhanced memory rates regardless of the time and brain region of the photostimulation. In contrast, the effect was only observed when the flash was directed to the mushroom bodies immediately after training. The observation that glutamate uncaging in the antennal lobes does not induce the same effect as it does when directed to the MBs gives support for a local effect of the uncaged glutamate and provides evidence against the possibility that glutamate has acted far away from the photostimulated region.

Previous studies of the role of glutamate in insect memory were based on systemic application of drugs whose specificity of action are described in vertebrates. Jaffe and Blanco (1994) reported an impairment in learning and long-term memory regarding operant conditioning in crickets when the NMDA receptor antagonist APV was injected before training. Maleszka et al. (2000) and Si et al. (2004) combined the use of glutamate reuptake blockers and NMDAR antagonists in bees and suggested that glutamate plays a role in long-term memory recall. Lopatina et al. (2002) tested agonists and antagonists of ionotropic glutamate receptors in honeybees and reported a modulation of memory $1 \mathrm{~min}$ after training and no effect $3 \mathrm{~h}$ later. Although all of these studies suggest that glutamate affects memory in insects, they lead to different interpretations regarding the precise role of glutamate in the memory process. The different interpretations are probably consequences of the still unknown specificity and kinetics of the drugs in insects and the possible side effects of the systemic administration. In the present work, we bypassed these problems by using local release of glutamate in defined brain regions. Local uncaging of glutamate in the mushroom bodies immediately after a weak training protocol enhances memory retention $2 \mathrm{~d}$ after training but has no effect on early memory at $2 \mathrm{~h}$. This result suggests that glutamate participates close to the time of training, and its action is critical for the induction of a late memory phase.

In honeybees, as in other models, the strength of training determines the induction of different memory phases (Müller, 2000). In harnessed bees, a single-trial conditioning induces the formation of a memory that decays over several days. Multipletrial conditioning induces a sequence of memory phases including a median-term memory $(<1 \mathrm{~d})$ (Grünbaum and Müller, 1998), a translation-dependent early LTM (1-2 d), and a transcription-dependent late LTM ( $\geq 3$ d) (Friedrich et al., 2004). Because memory performance in vehicle and glutamate groups (Fig. 2B) resembles the memory retention curves induced by single- and multiple-trial training, respectively, it is tempting to speculate that release of glutamate in the mushroom bodies mechanistically converts a weak into a strong training and thus induces LTM. However, because different memory phases are induced in parallel and subserved by different molecular pathways (Grünbaum and Müller, 1998; Friedrich et al., 2004), it is also likely that glutamate release induced the formation of a not yet described late-memory phase. In the latter case, uncaging glutamate may improve memory after multiple-trial conditioning. This, however, has not been tested, because multiple-trial training induces a very high memory level (80-100\%), which would not be distinguished from an additional effect of glutamate uncaging after multiple-trial conditioning.

The demonstrated function of glutamate in the mushroom bodies will be the basis of future experiments to identify the signaling cascades underlying the formation of memory. This will be of special importance, because, in contrast to the extensive knowledge of mushroom body function in Drosophila learning (Davis, 2005), there is only little information on signaling cascades critical for learning and memory formation in the honeybee mushroom bodies. Although the cAMP-dependent protein kinase A (PKA), a central player in LTM formation throughout the animal kingdom, shows a high expression in Drosophila and honeybee mushroom bodies (Müller, 1997), its role in mushroom bodies has been demonstrated convincingly only in Drosophila. In contrast, in the honeybee, the PKA activity in the antennal lobes, another brain area implicated in associative olfactory learning (Hammer and Menzel, 1998), has been demonstrated to be required for induction of late-memory phases (Müller, 2000). However, memory impairment by systemic blocking of PKA activity (Müller, 2000) and downregulation of the PKA catalytic subunit (Fiala et al., 1999) that is partially restricted to the mushroom bodies, suggests a function of the PKA in the mushroom body, as reported previously in Drosophila. Thus, the PKA cascade in the mushroom bodies is a potential signaling cascade that may mediate the glutamate function with respect to memory formation.

Although our work provides no information with respect to the type of glutamate receptor that participates in memory formation, a recent work in genetically manipulated Drosophila reported a function of NMDAR for memory formation of an aversive operant conditioning (Xia et al., 2005). In contrast to our observations, the NMDAR in Drosophila also affects short-term memory (STM). Multiple-trial training overcomes the deficit in STM but not in LTM, pointing to an implication of the Drosophila NMDAR in different aspects of learning and memory formation. Although other possibilities remain, this difference regarding STM could be explained by the contribution of distinct brain areas, because the manipulated NMDAR is weakly expressed throughout the brain with higher expression levels in cell bodies surrounding the calyces of the MBs. The discrepancy between the localization of the Drosophila NMDAR (around the calyces) and the effect obtained using uncaging of glutamate in the honeybee mushroom bodies suggest that other glutamate receptors re- 
ported to be expressed in the insect mushroom bodies (Volkner et al., 2000; Funada et al., 2004) may also participate in the glutamate function in memory formation in honeybees.

In summary, our results obtained with honeybees, together with the recent report on Drosophila, provide independent evidence for the role of glutamate in memory formation in insects; this data furthermore supports the notion that glutamatergic neurotransmission is a conserved key element in neural plasticity, as demonstrated previously for other species and phyla (Troncoso and Maldonado, 2002; Riedel et al., 2003; Roberts and Glanzman, 2003).

\section{References}

Bicker G, Schafer S, Ottersen OP, Storm-Mathisen J (1988) Glutamate-like immunoreactivity in identified neuronal populations of insect nervous systems. J Neurosci 8:2108-2122.

Bitterman M, Menzel R, Fietz A, Schäfer S (1983) Classical conditioning of proboscis extension in honeybees (Apis mellifera). J Comp Psychol 97:107-119.

Callaway EM, Yuste R (2002) Stimulating neurons with light. Curr Opin Neurobiol 12:587-592.

Cayre M, Buckingham SD, Yagodin S, Sattelle DB (1999) Cultured insect mushroom body neurons express functional receptors for acetylcholine, GABA, glutamate, octopamine, and dopamine. J Neurophysiol 81:1-14.

Connolly JB, Roberts IJ, Armstrong JD, Kaiser K, Forte M, Tully T, O’Kane CJ (1996) Associative learning disrupted by impaired Gs signaling in Drosophila mushroom bodies. Science 274:2104-2107.

Crowther J (2001) The ELISA guidebook. Totowa, NJ: Humana.

Davis RL (2005) Olfactory memory formation in Drosophila: from molecular to systems neuroscience. Annu Rev Neurosci 28:275-302.

de Belle JS, Heisenberg M (1994) Associative odor learning in Drosophila abolished by chemical ablation of mushroom bodies. Science 263:692-695.

Fiala A, Müller U, Menzel R (1999) Reversible downregulation of protein kinase A during olfactory learning using antisense technique impairs long-term memory formation in the honeybee, Apis mellifera. J Neurosci 19:10125-10134.

Friedrich A, Thomas U, Müller U (2004) Learning at different satiation levels reveals parallel functions for the cAMP-protein kinase A cascade in formation of long-term memory. J Neurosci 24:4460-4468.

Funada M, Yasuo S, Yoshimura T, Ebihara S, Sasagawa H, Kitagawa Y, Kadowaki T (2004) Characterization of the two distinct subtypes of metabotropic glutamate receptors from honeybee, Apis mellifera. Neurosci Lett 359:190-194.

Grünbaum L, Müller U (1998) Induction of a specific olfactory memory leads to a long-lasting activation of protein kinase $\mathrm{C}$ in the antennal lobe of the honeybee. J Neurosci 18:4384-4392.

Hammer M, Menzel R (1998) Multiple sites of associative odor learning as revealed by local brain microinjections of octopamine in honeybees. Learn Mem 5:146-156.

Heisenberg M, Borst A, Wagner S, Byers D (1985) Drosophila mushroom body mutants are deficient in olfactory learning. J Neurogenet 2:1-30.

Isabel G, Pascual A, Préat T (2004) Exclusive consolidated memory phases in Drosophila. Science 304:1024-1027.

Jaffe K, Blanco ME (1994) Involvement of amino acids, opioids, nitric oxide and NMDA receptors in learning and memory consolidation in crickets. Pharmacol Biochem Behav 47:493-496.

Jan LY, Jan YN (1976) L-Glutamate as a neurotransmitter at the neuromuscular junction. J Physiol (Lond) 262:215-236.

Kucharski R, Ball E, Hayward D, Maleszka R (2000) Molecular cloning and expression analysis of a cDNA encoding a glutamate transporter in the honeybee brain. Gene 244:399-405.

Lopatina N, Ryzhova I, Chesnokova E (2002) The role of non-NMDAreceptors in the process of associative learning in the honeybee Apis mellifera. J Evol Biochem Physiol 38:211-217.

Maleszka R, Helliwell P, Kucharski R (2000) Pharmacological interference with glutamate re-uptake impairs long-term memory in the honeybee Apis mellifera. Behav Brain Res 115:49-53.

Müller U (1997) Neuronal cAMP-dependent protein kinase type II is concentrated in mushroom bodies of Drosophila melanogaster and the honeybee, Apis mellifera. J Neurobiol 33:33-44.

Müller U (2000) Prolonged activation of cAMP dependent protein kinase during conditioning induces long term memory in honeybees. Neuron 27:159-168.

Parmentier M, Pin J, Bockaert J, Grau Y (1996) Cloning and functional expression of a Drosophila metabotropic glutamate receptor expressed in the embryonic CNS. J Neurosci 16:6687-6694.

Pascual A, Preat T (2001) Localization of long-term memory within the Drosophila mushroom body. Science 294:1115-1117.

Riedel G, Platt B, Micheau J (2003) Glutamate receptor function in learning and memory. Behav Brain Res 140:1-47.

Roberts AC, Glanzman DL (2003) Learning in Aplysia: looking at synaptic plasticity from both sides. Trends Neurosci 26:662-670.

Schürmann F, Ottersen O, Honegger H (2000) Glutamate-like immunoreactivity marks compartments of the mushroom bodies in the brain of the cricket. J Comp Neurol 418:227-239.

Seal RP, Daniels GM, Wolfgang WJ, Forte MA, Amara SG (1998) Identification and characterization of a cDNA encoding a neuronal glutamate transporter from Drosophila melanogaster. Receptors Channels 6:51-64.

Si A, Helliwell P, Maleszka R (2004) Effects of NMDA receptor antagonists on olfactory learning and memory in the honeybee (Apis mellifera). Pharmacol Biochem Behav 77:191-197.

Sinakevitch I, Farris SM, Strausfeld NJ (2001) Taurine-, aspartate- and glutamate-like immunoreactivity identifies chemically distinct subdivisions of Kenyon cells in the cockroach mushroom body. J Comp Neurol 439:352-367.

Troncoso J, Maldonado H (2002) Two related forms of memory in the crab Chasmagnathus are differentially affected by NMDA receptor antagonists. Pharmacol Biochem Behav 72:251-265.

Ultsch A, Schuster CM, Laube B, Betz H, Schmitt B (1993) Glutamate receptors of Drosophila melanogaster. Primary structure of a putative NMDA receptor protein expressed in the head of the adult fly. FEBS Lett 324:171-177.

Volkner M, Lenz-Bohme B, Betz H, Schmitt B (2000) Novel CNS glutamate receptor subunit genes of Drosophila melanogaster. J Neurochem 75:1791-1799.

Wieboldt R, Gee KR, Niu L, Ramesh D, Carpenter B, Hess G (1994) Photolabile precursor of glutamate: synthesis, photochemical properties, and activation of glutamate receptors on a microsecond time scale. Proc Natl Acad Sci USA 91:8752-8756.

Xia S, Miyashita T, Fu TF, Lin WY, Wu CL, Pyzocha L, Lin IR, Saitoe M, Tully T, Chiang AS (2005) NMDA receptors mediate olfactory learning and memory in Drosophila. Curr Biol 15:603-615. 